\title{
PRIMER REGISTRO DEL TIBURON PERRO CENTROSCYLLIUM NIGRUM (CHONDRICHTHYES: SQUALIDAE) EN AGUAS DEL PACIFICO COLOMBIANO
}

\author{
FIRST RECORD OF DOGFISH SHARK CENTROSCYLLIUM \\ NIGRUM (CHONDRICHTHYES: SQUALIDAE) IN THE COLOMBIAN \\ PACIFIC OCEAN
}

\author{
Efraín A. Rubio² ${ }^{2}$ Milton J. Pedraza ${ }^{1}$ \& Luis A. Zapata ${ }^{3}$ \\ ${ }^{1}$ Universidad del Valle, Departamento de Biología, Cali (Colombia). \\ ${ }^{2}$ Universidad de Concepción, Maestría en Ciencias Mención en Pesquerías (Chile). E-mail: mipedraz@udec.cl \\ ${ }^{3}$ WWF Colombia. Programa marino - costero. E-mail: lazapata@wwf.org.co
}

\begin{abstract}
RESUMEN
El tiburón perro, Centroscyllium nigrum Garman, 1899, es un representante pequeño de la familia Squalidae que habita en aguas profundas del Pacífico Oriental. Se registra por primera vez un ejemplar hembra adulta en el Pacífico colombiano, capturado mediante trampas en la pesquería del camarón cabezón Heterocarpus hostilis Faxon, 1893, lo cual constituye un importante hallazgo para el área entre el Golfo de Panamá e Islas Galápagos, confirmado su presencia en esta zona.
\end{abstract}

Palabras claves: Primer registro, Centroscyllium nigrum, Pacífico colombiano.

\section{ABSTRACT}

The coombtooth dogfish, Centroscyllium nigrum Garman 1899, is a small, deepwater squaloid shark that live in Eastern Pacific. One adult female specimen of $C$. nigrum, reported for the first time in Colombian Pacific Ocean a specimen was captured by mean trap in fisheries shrimp big- headed Heterocarpus hostilis Faxon, 1893. These report confirms the presence in the zone. This report confirm the presence of this specie in the zone between Panama Gulf and Galapagos Island.

Keywords: First record; Centroscyllium nigrum; Colombian Pacific Ocean.

\section{INTRODUCCION}

Los miembros de la familia Squalidae (Pisces: Chondrichthyes: Squaliformes) son tal vez los tiburones de mayor diversidad específica, distribución geográfica y batimétrica del mundo; sus especies pueden vivir desde zonas articas y antárticas hasta zonas tropicales y subtropicales, a profun-didades de hasta $6.000 \mathrm{~m}$ (Compagno 1984); algunas de éstas presentan gran interés para la pesca en muchos países.
Actualmente son conocidos 17 géneros y 67 especies; con géneros cuyas especies alcanzan tamaños tan pequeños como Squaliolus y Euprotomicrus que logran la madurez sexual a tallas menores de $20 \mathrm{~cm}$ de longitud, en contraste con gigantes de zonas epibentónicas de mares boreales como los del género Somniosus con más de 6 metros de longitud (Compagno 1984). Del género Centroscyllium en la actualidad se conoce ocho especies Bigelow \& Schroeder (1948), reconocieron cinco de ellas; posteriormente Abe (in Campagno 1984) agrega una 
más y recientemente Nakaya (in Okamura et al.1982) reporta dos posibles nuevas especies en cercanías de Japón. Este género es un habitante frecuente de zonas tropicales y subtropicales del mundo; su captura se torna particularmente difícil por los rangos de profundidad que habitan entre 400 y $1.600 \mathrm{~m}$. Vale la pena aclarar que Compagno, (1999) ubica esta especie en la familia Etmopteridae con 5 géneros y 38 especies descritas ( 2 sin confirmar), de las cuales actualmente se conocen siete especies del género Centroscyllium.

Centroscyllium nigrum es una especie netamente de profundidad, de ella se tiene poco conocimiento de sus parametros de vida (alimentación, reproducción, comportamiento, etc). Garman (1899), al describir esta especie la cita frente a Panamá; mas tarde Bigelow \& Schroeder (1957) igualmente la reportan para el Pacífico tropical, frente a América Central y en la zona de hawaii. Campagno (1984) ya la cita para mayores latitudes frente a Chile zona centro-norte. La captura de este espécimen de Centroscyllium nigrum en el sur del Pacífico colombiano constituye un importante hallazgo para el área entre el Golfo de Panamá e Islas Galápagos, confirmando su presencia en esta zona, ya que Rubio (1987) señala su probable existencia, pero aclara no poseer material recolectado.

\section{MATERIALES Y METODOS}

La recolecta del ejemplar analizado se realizo a bordo de la embarcación coreana "Sam Hae 101", cuya pesquería objetivo es el camarón cabezón de profundidad Heterocarpus hostilis, utilizandocomo arte trampas metálicas recubiertas con malla, con carduma Cetengraulismysticetus Günther, 1866 como carnada, las cuales llegan a profundidades entre 600 y 900 m con una duración promedio en el agua de 13 horas. Después de la captura el ejemplar se fijó en formalina al $10 \%$ para su traslado al laboratorio de Ictiología de la sección de Biología Marina de la Universidad del Valle.

La identificación del ejemplar se realizó utilizando las claves y descripciones de Chirichigno (1974, 1978); Compagno (1984) y Meléndez \& Meneses (1989). Los datos morfométricos están dados en milímetros y expresados en porcentajes de la longitud total (Lt) (Tabla I). El ejemplar se conservó en alcohol y depositó en la colección de Ictiología de referencia de la sección de Biología Marina de la Universidad del Valle (CIRUV) con número de catálogo 95001 en la ciudad de Cali (Colombia).

\section{RESULTADOS}

Sinonimia: Centroscyllium ruscosum Gilbert 1905. Nombres vernaculares: Castellano: Tiburón perro. Inglés: Combtooth dogfish.

Material EXAMINADo: 1 ejemplar hembra (ㅇ) de 334 $\mathrm{mm}$ de longitud total (figura 1), recolectado el 10 de julio de 1995 . Lance $\mathrm{N}^{\circ} 12$ posición geográfica inicial $\left(1^{\circ} 47^{\prime} 02^{\prime} \prime \mathrm{N}-79^{\circ} 30^{\prime} 87 \mathrm{~W}\right)$, final $\left(1^{\circ} 48^{\prime} 57^{\prime}\right.$ ' N - 79 $27^{\prime} 93^{\prime}$ 'W), a $850 \mathrm{~m}$ de profundidad. Registro de catálogo (CIRUV-95001). Colector: Biólogo Milton Pedraza.

CARACTERES Distintivos: los conteos y las medidas proporcionales se presentan en la Tabla I. El ejemplar analizado se caracteriza por poseer un cuerpo moderadamente robusto y corto, dos aletas dorsales ambas con espinas (segunda de mayor tamaño), ausencia de aleta anal, pedúnculo caudal corto, segunda aleta dorsal inserta por detrás del inicio de la aleta pélvica, aleta pélvica ubicada en el tercio posterior del cuerpo, aletas pectorales con borde anterior redondeado se insertan por detrás de la última abertura branquial, aberturas branquiales de longitudes variables, boca ampliamente arqueada, dientes tricúspides en ambas mandíbulas, cúspide central mayor que las laterales, cúspides laterales de tamaño semejante, cuerpo recubierto de dentículos dérmicos, dentículos de la zona cefálica presentan base estrellada con una espina central ligeramente curvada hacia atrás, dentículos del cuerpo de igual configuración que los de la cabeza pero su espina central mas inclinada hacia atrás, color oscuro uniforme excepto en los márgenes de las aletas dorsales y pectorales que son levemente más claras.

Distribución Geográfica : inicialmente Garman (1989) realiza la descripción de la especie con individuos registrados fuera del Golfo de Panamá. Más tarde Bigelow \& Schroeder (1957) reportan especímenes para el Pacífico tropical frente a América Central y en la zona de Hawai. Registrada también por Bearez (1996) para el Ecuador, actualmente esta especie es reconocida para el Pacífico Oriental y Central (Islas Hawai, Sur de California, Panamá, Isla de los Cocos, Chile e Islas Galápagos) en Compagno (1984). La localización de nuestro reporte concuerda con el área de distribución citada anteriormente. 
Centroscyllium nigrum en el Pacífico colombiano: RuBIo E. ET AL.

TABLA I. Análisis morfométrico y merístico del ejemplar hembra Centroscyllium nigrum reportado para la costa pacífica colombiana.

TABLE I. Morfometric and measurements analysis of individual female Centroscyllium nigrum reported for pacific coast colombiana.

\begin{tabular}{|c|c|c|}
\hline MEDIDA & LONGITUD (mm) & PORCENTAJE EN L \\
\hline Longitud total & 334 & \\
\hline Altura del cuerpo & 37 & 11,1 \\
\hline Altura del pedúnculo caudal & 9 & 2,7 \\
\hline Altura de la cabeza & 47 & 14,1 \\
\hline Altura del ojo & 10 & 3 \\
\hline Ancho de la boca & 30 & 9 \\
\hline Longitud de la cabeza & 80 & 24 \\
\hline Longitud de la boca & 10 & 3 \\
\hline Longitud internarinal & 15 & 4,5 \\
\hline Longitud pre-orbital & 19 & 4,8 \\
\hline Longitud pre-espiracular & 39 & 11,7 \\
\hline Longitud espiráculo & 6 & 1,8 \\
\hline Longitud ojo-espiráculo & 5 & 1,5 \\
\hline Longitud pre-branquial & 60 & 18 \\
\hline Longitud interbranquial & 2 & 0,6 \\
\hline Longitud $1^{\mathrm{a}}$ abertura branquial & 14 & 4,2 \\
\hline Longitud $2^{\mathrm{a}}$ abertura branquial & 12 & 3,6 \\
\hline Longitud $3^{\text {a }}$ abertura branquial & 11 & 3,3 \\
\hline Longitud $4^{\mathrm{a}}$ abertura branquial & 10 & 3 \\
\hline Longitud $5^{\mathrm{a}}$ abertura branquial & 10 & 3 \\
\hline Longitud pre-pectoral & 81 & 24,3 \\
\hline Longitud pre- pélvica & 180 & 53,9 \\
\hline Longitud de la $1^{\text {a }}$ dorsal & 47 & 14,1 \\
\hline Longitud aleta pélvica & 41 & 12,3 \\
\hline Altura aleta pélvica & 7 & 2,1 \\
\hline Altura de la pectoral & 30 & 9 \\
\hline Espacio $2^{\mathrm{a}}$ dorsal-caudal & 30 & 9 \\
\hline Espacio pélvica-caudal & 44 & 13,2 \\
\hline Margen anterior de la $1^{\mathrm{a}}$ dorsal & 40 & 12 \\
\hline Margen posterior de la $1^{\mathrm{a}}$ dorsal & 9 & 2,7 \\
\hline Base de la $1^{\text {a }}$ dorsal & 30 & 9 \\
\hline Base de la pectoral & 9 & 2,7 \\
\hline Margen anterior de la pectoral & 28 & 8,4 \\
\hline Margen dorsal de la caudal & 77 & 23,1 \\
\hline
\end{tabular}




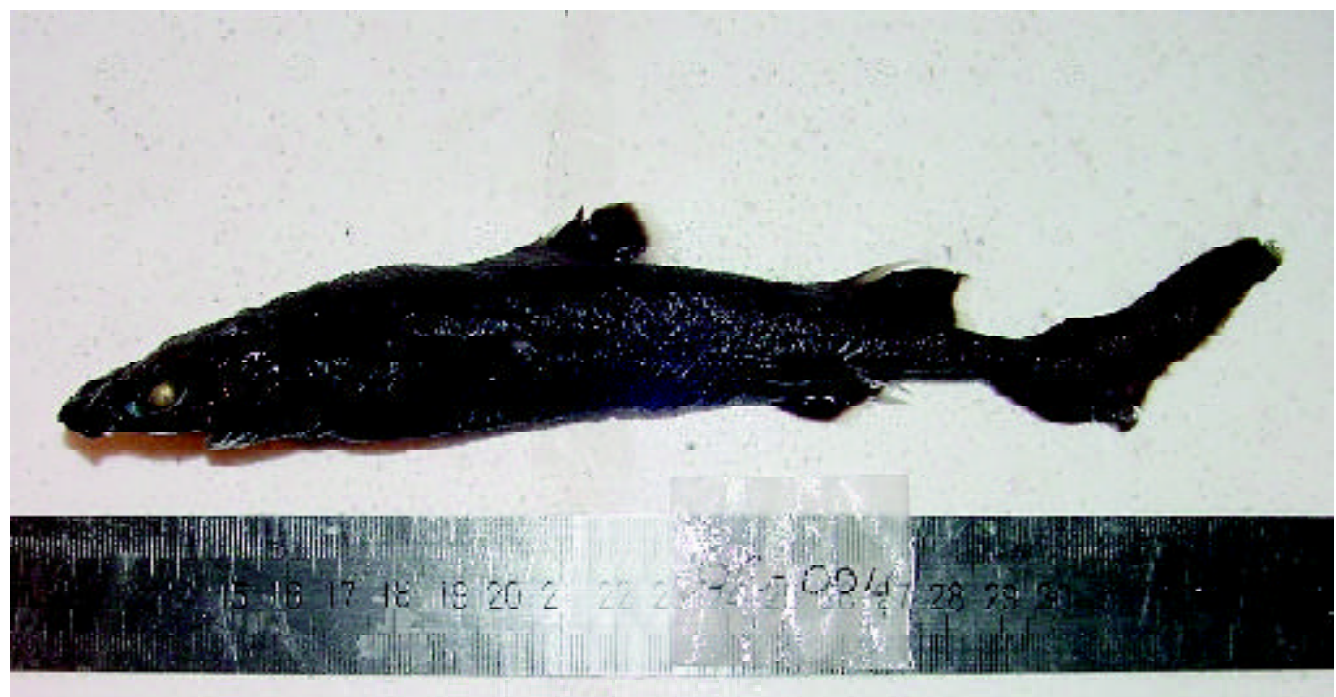

FIGURa 1. Individuo adulto de Centroscyllium nigrum. Número de catalogo CIRUV 95001. Sección Biología Marina. Universidad del Valle.

FIGURE 1. Adult of Centroscyllium nigrum. Report number CIRUV 95001. Marine biology section. University of Valle

TALlAs: Campagno etal. (1995) argumentan una longitud máxima alcanzada por individuos de esta especie de $500 \mathrm{~mm}$ de L; describiendo a las hembras como más grandes, alcanzando con facilidad esta talla máxima. Los machos adultos suelen no sobrepasar los $400 \mathrm{~mm}$ de longitud. Nuestro ejemplar de $334 \mathrm{~mm}$ es una hembra en estado adulto.

Hábitat y Biología: Son pocos los conocimientos sobre aspectos de esta especie y no se pudieron obtener datos de alimentación o reproducción de nuestro ejemplar; Eschmeyer et al. (1993) señalan que los tiburones de esa familia principalmente consumen peces, crustáceos y cefalópodos; igualmente establecen que son ovovivíparos. Se resalta también que fue recolectado entre $850 \mathrm{~m}$ de profundidad, sobre fondos fangosos a temperaturas de $3-4^{\circ} \mathrm{C}$

Fauna Acompañante: La fauna que también fue capturada incidentalmente en las faenas de pesca del camarón de aguas profundas, en la zona que se halló el tiburón perro, está compuesta por las familias de peces Nemichthyidae, Uranoscopidae, Congridae, Macrouridae, Ogcocephalidea y Zoarcidae.

\section{DISCUSION}

Los primeros registros de esta especie se originaron en el Pacífico central Oriental, donde Garman (1899) recolectó cuatro especímenes a profundidades entre 999 y 1016 metros, siendo uno de ellos el holotipo que utilizó para su primera descripción. Posteriores reportes en esta misma zona corresponden a los realizados por Bearez (1996) y Bigelow \& Schroeder (1957), todos en localidades fuera de las delimitaciones del Pacífico colombiano. Si se toman en consideración y se georreferencia las dos localidades de la recolección presentadas por Garman (1899) en la expedición "albatros" $\left(7^{\circ} 09^{\prime} 30^{\prime} \mathrm{N}-81^{\circ} 08^{\prime} 30^{\prime}\right.$ W y $6^{\circ} 30^{\prime} \mathrm{N}-81^{\circ} 44^{\prime} \mathrm{W}$ ), éstas se sitúan en aguas fuera del Golfo de Panamá, pero pertenecientes dentro de las limitaciones internacionales de fronteras marítimas a ese país, aclarando la confusión actual de que estos registros estarían dentro del Pacífico colombiano. De tal manera que el presente trabajo además de reafirmar la presencia de esta especie en el Pacífico Tropical Americano, se constituye en el primer registro para aguas de la costa pacífica colombiana, ya que Rubio (1987) señala su probable presencia, pero aclara no poseer material recolectado en esta área. 
Este trabajo contribuye en los aspectos biogeográficos de la especie, ya que confirma su presencia dentro de la distribución geográfica sugerida en Compagno (1984). Y es, a la vez, una contribución importante para el conocimiento de las especies marinas costeras de aguas profundas de nuestro país.

\section{AGRADECIMIENTOS}

Se agradece al Instituto Nacional de Pesca y Acuicultura INPA, por la obtención de los ejemplares dentro del proyecto "Evaluación del camarón de profundidad (Heterocarpus spp.) En el Pacífico Colombiano". Al Departamento de Biología de la Universidad del Valle, por la utilización de sus instalaciones y a tres evaluadores anónimos que entregaron sus apreciaciones a este trabajo.

\section{BIBLIOGRAFIA}

Bearez, P.H. 1996. Listas de los peces marinos del Ecuador. Revista Biología Tropical 44(2): 731-741.

Bigelow, H.B. \& W.C. Schroeder. 1948. Sharks. In: J. Tee-Van, C. M. Breder, S. F. Hildebrand, A. E. Parr and W. C. Schoreder (eds.). Fishes of the western North Atlantic. Sears Found. Mar. Res. Mem., $\mathrm{N}^{\circ} 1: 59-576$

Bigelow, H.B \& W.C. Schroeder. 1957. A study of the sharks of the suborden Squaloidea. Bull. Mus. Comp. Zool., 117(1): 1-150.

Chirichigno, F.N. 1974. Clave para identificar los peces marinos del Perú. Bol. $\mathrm{N}^{\circ}$ 44. Instituto del Mar del
Perú- IMARPE. Callao. 387 pp.

. 1978. Nuevas adiciones a la Ictiofauna marina del Perú. Informe $\mathrm{N}^{\circ}$ 46. Instituto del Mar del PerúIMARPE. Callao. 109 pp.

Compagno, L.J.V. 1984. Sharks of the world. An annotated and illustrated catalogues of shark species known to date. Part 1. Hexanchiformes to Lamniformes. FAO species catalogue. FAO Fish. Synop., Vol. 4: $249 \mathrm{p}$

Compagno, L.J.V., F. Krupp \& W. Schneider. 1995. Tiburones. In W. Fischer, F. Krupp, W. Schneider, C. Sommer, K.E. Carpenter \& V. Niem (eds.). Guía FAO para la identificación de especies para los fines de la pesca. Pacífico Centro-Oriental. FAO, Roma. Vol. 3:647-744.

Eschmeyer, W., E. Herald \& H. Hammann. 1983. A field guide to Pacific coast fishes of the North America. Houghton mifflin, Boston. $336 \mathrm{p}$.

Garman, S. 1899. Reports of an exploration of the west coasts of Mexico, Central and South America and the Galapagos Islands, in charge of Alexander Agassiz, by the U.S. Fish Commission Steamer "Albatross", during 1891, Lieut. Cmdr. Z. L. Tanner, U. S. N. Commanding. XXVI: The fishes. Memoriam. Museum Comp Zoological Harvard. College. 24:1-431.

Meléndez. R.C. \& D.R. Meneses. 1989. Tiburones del talud continental recolectados entre Arica $\left(18^{\circ}\right.$ 19'S) e Isla Mocha (38 30' S), Chile. Investigaciones Marinas Valparaíso 17: 3-73.

Okamura, O., K. Амaока. \& F. Mitani. 1982. Fishes of the Kyushu-Palau ridge and Tosa Bay Japan. Fisheries Research Conservation Association. Tokyo. $435 \mathrm{p}$.

Rubio, E.A. 1987. Lista sistemática de peces costeros y de profundidad del Pacífico colombiano. Centro de Publicaciones, Facultad de Ciencias, Departamento de Biología Marina de la Universidad del Valle. $112 \mathrm{pp}$. 